\title{
EDITORIAL
}

\section{Insulin Resistance, Hyperinsulinemia and COVID-19 Mortality}

Syed Muhammad Imran Majeed, Rehma Ahsan Gilani

Insulin resistance is at the heart of pathogenesis of major noncommunicable disorders namely atherosclerosis, hypertension, diabetes mellitus, obesity, even autoimmune diseases, possibly allergies and some cancers. Inflammation as instigated by hyperinsulinemia appears responsible for a major portion of noncommunicable diseases burden. The modern human diet wherein most of the calories come from starches, is the singular most important prevalent cause of hyperinsulinemia and insulin resistance. Most of the humanity is either diabetic or prediabetic (glucose intolerant) and vulnerable to dangerous consequences of hyperinsulinemia and insulin resistance.

One such consequence that we are faced with today, is mortality in COVID-19 infected patients who suffer from diabetes mellitus.

Very little, certainly inadequate, attention has been paid to hyperinsulinemia and its life threatening repercussions during the ongoing COVID-19 pandemic.

Attention of researchers and health care systems is drawn to the obvious dangers of this oversight; be that the ongoing mortality due to COVID-19, or the noncommunicable disease burden in general.

\section{Editor-in-Chief}

How to cite this: Majeed SMI, Gilani RA. Insulin Resistance, Hyperinsulinemia and COVID-19 Mortality. Life and Science. 2020; 1(suppl): 1-1. doi: http://doi.org/10.37185/LnS.1.1.178

This is an Open Access article distributed under the terms of the Creative Commons Attribution License (http://creativecommons.org/licenses/by/4.0), which permits unrestricted use, distribution, and reproduction in any medium, provided the original work is properly cited 